Simultaneous dislocation of both wrists has been, $I$ believe, reported to have occurred in a few instances; but I have no references to cite, and should be inclined to question the correctness of the diagnosis, in view of the great rarety of the undoubted examples of displacement of that joint.

\section{EXTERNAL MEDIAN PERIN EAL URETHROTOMY, FOR CYSTITIS AND FOR THE REMOVAL OF MORBID GROWTHS FROM THE BLADDER.}

BY B. B. BONTACOL, M.D.

[Read before the Surgical Section of the American Medical Association, June, $x 88_{3}$.]

The operation is a median incision of the perinaum, commencing about one inch from the anus, and extending about one or one and a half inches towards the scrotum, according to the depth of the perinæum and size of the subject, and terminating in the membranous portion of the urethra, which should be laid open just in front of the bladder to the extent of one-half to three-fourths of an inch. This is done on a grooved staff, while the patient is in the lithotomy position, the bowels having previously been emptied. The operation is simple, almost bloodless, and rapidly and easily executed. The finger may then be admitted to the bladder, and by counter pressure above the pubes (if the patient is profoundly etherized) every part of the viscus may be made to touch the point of the finger, thereby obtaining most intelligent and thorough examination of the whole interior, and enabling the operator to remove with spoon, forceps or snare any morbid growth capable of being removed. The operation is especially adapted to cases of irritable bladder from vegetation or polypoid growths, and to cases of chronic cystitis from other causes, that have proved incurable by drugs and hygienic methods. The lateral operation, as for lithotomy, was in November, $185^{\circ}$, practiced by Prof. Parker, of New York, for the relief of an obstinate case of cystitis, the result of gonorrhoal inflammation, but this method, much more simple and less dangerous, has not been practiced for this class of cases, I believe, until Sir Henry Thompson, of London, in 1882 , recommended and practiced it on a few cases with gratifying results, and his publications induced me to apply it to two very bad cases that came under my care, one of which, F. S., a young man of 28 years, of correct habits and specific history, and who was a strong laboring man, a moulder by occupation, until March, I882, when he took sick with rheumatism, which lasted two months; and during the sickness his bladder trouble commenced, with scalding of the urine, accompanied by pus and mucus in large quantities. Efforts to expel this brought on distressing tenesmus and protrusion of the bowel, and he was obliged to void his urine every half hour night and day, and often the burning in the penis and straining were so unbearable that he was forced to cry aloud. He came under my care in October, 1882 . Irrigation of the bladder and numerous other means were tried, but it was so painful to him that he would not submit to it with any regularity, and then only when under the effects of an anodyne injection of one or two teaspoonfuls of laudanum. Hyosciamus and camphor, salicylate of soda, bromide of potassium, buchu, uva ursa and epigea repens, balsam copaiba, turpentine and other remedies were given internally, while laudanum injections, opii suppositories and ergot were used by the rectum, and the bladder was washed out whenever he could tolerate it with solutions of borax and carbolic acid, chlorate of potassa, bi-carb. soda, permanganate of potassa, alum and sulph. zinci, with varying degrees of relief. On March 20, I883, performed this operation, and found two polypoid growths hanging from the upper and anterior surface of the bladder, which I removed with the finger nail and forceps without producing much hæmorrhage. Bladder was thoroughly washed out, and a drainage tube of large caliber inserted and left in. The tube was retained with some difficulty, owing to the severe contraction of the viscus, but its presence was not painful to the patient, and through this his bladder was two or three times daily irrigated with solution of borax and carbolic acid, and the slime and pus escaped as fast as secreted. The agonizing burning pain in the penis ceased at once, and returned only on a few occasions, when the tube had become obstructed or had slipped down into the perinæal wound. He could sleep a greater part of the night without anodynes, and expressed himself as quite happy compared with his former state. About the middle of April both lower limbs swelled and all the joints were painful, which appeared as a form of rheumatism, and yielded to the internal use of salicylate of sodium. 'This man is now well enough to walk some distance daily, and his general health nearly restored to normal standard. This drainage tube was kept in use one month, since which time it has not been required, and the bladder empties itself mostly through the natural passage, but occasionally some escapes from the sinus, which is not yet entirely healed.

CASE No. 2.-I. H. N., moulder by occupation, 26 years of age, with no specific history, who for 3 or 4 years had been suffering from irritable bladder, took cold about 8 months ago and had a decided aggravation of his troubles, and 8 months ago married, soon after which he became much worse and was obliged to remain in his bed, being unable to walk on account of the painful straining and burning pain in the penis. He was obliged from this time to pass his urine every hour or oftener day and night and always accompanied by quantities of mucus and pus. He was admitted to St. Peter's Hospital at Albany, New York, and remained there ten weeks, and finding no relief returned to his home in West Troy, and came under my care April 4th, I883. Irrigation of his bladder had been practiced by himself and attendants since January, $188_{3}$, but not regularly on account of the great pain attending the introduction of the catheter, and often suffering four or five hours torment after the operation. I did not succeed satisfactorily with the use of internal remedies, and May 7 th, I 883 , I performed median external perinæal urethrotomy and found just within the neck of the bladder on his left side a cock's comb-shaped vegetation, which I removed with forceps and my 
finger nails, and inserted a drainage tube doubled upon itself, with two eyes in the inner curve of the doubles, and kept in the form of an open loop by a slender brass wire of sufficient temper to keep the knuckl: of tube expanded within the bladder, this I found to be self-retaining, and has great advantage over the straight tubes in retaining its position without tying; the loop was curved downward so that it would lay in the cul-de-sac behind the neck and siphon off the morbid secretions disposed to collect there. Through this double tube his attendant easily irrigated the bladder from a fountain syringe without pain to the patient, and the relief this man experienced was, as he expressed it, charming. He was wasted to a great degree by his long suffering, but soon regained his appetite and enjoyed sleep at night for hours, whereas formerly he was every few minutes disturbed, and his suffering from the burning pain and tenesmus was pitiable, notwithstanding the use of liberal opiates by the rectum. All that ceased and has not returned, and is now dispensing with the tubes and able to sit $\mathrm{ap}$ and walk about the rooms. The sinus still discharges urine, but the mucus and other evidences of inflammation have gone.

A singular accident occurred in the treatment of this case. A fortnight after the operation he was taken with a smart hæmorrhage which was controlled by use of cold alum solution and sub. sulph. of iron, which latter ingredient I should advise not to be again used, as the coagulum formed in the bladder was so hard that I was compelled to break it up and remove it piece-meal with the lithotomy forceps. I hope to be able to report shortly two of the cases of the same trouble, in which I intend to perform the operation.

\section{GERMICIDES,-AN EXPERIMENTAL STUDY UPON THE COMPARATIVE VALUES OF ANTISEPTICS.}

BY HENRY O. MARCY, M.D., BOSTON, MASS.

[Read to the Section on Surgery and Anatomy.]

The deleterious effect of micro-organisms upon wounds can no longer be doubted. The so-called germ theory has passed into the realm of demonstrated fact. The patient labors of scores of very careful investigators during the last generation have slowly elaborated the fact that fermentation is dependent upon a particulate something, omnipresent in ordinary surroundings, and clearly shown that this something belongs to one or another of the varieties of exceedingly minute vegetable organisms, which compensate by number and rapidity of development that which they lack in size. In the study of these ninute organisms the important question arises, perhaps second at present to none in the entire realm of the science of metlicine, how can we be protected from their deleterious effects?

As the outcome of experimental research, there has been offered to the profession a large number of socalled germicides, and the thought which prompted the laboratory studies that form the basis of this paper was to test the comparative values of as large a number as the time at our disposal would permit. We take pleasure in acknowledging the painstaking and efficient aid of Dr. Samuel N. Nelson, of Cambridge, in the entire series of experiments.

At the outset it is well to bear in mind that one agent may possess a value dependent upon its power to destroy the vital organism, and another upon its power to restrain it from active development, although it has no destructive effect upon the organism itself. 'Thus, in a certain sense, as a result, this latter group of agents may be classed with the former, as, for example, chloride of zinc, which restrains the bacteria from development, because it acts chemically upon the albumen of the nutrient fluid.

Although the results of the experimental researches to be given, have an indirect bearing upon the medical value of the different agents tested, the primal thought which prompted the investigation was to ascertain the value which such agents may have in the1r surgical uses, for the destruction of organisms which cause putrefaction in wounds, the agent to be of value must act quickly, and it was therefore held as important that the material to be tested should have only a brief period of contact with the germbearing fluid. For this reason a considerable quantity, in each instance three drachms, of the test fluid was carefully mixed with one drachm of the putrefactive material and after a given measured time a small portion of the mixture, two or three drops only, transferred to the aseptic nutrient fluid which was carefully protected from contamination and placed under observation. If bacteria have preserved their vitality during the brief period of contact with the antiseptic, the minute quantity thus taken as seed will rapidly germinate in the nutrient solution and soon putrefactive changes will be apparent.

Thus we know that either the antiseptic was not of a sufficient strength, or not in contact for a length of time requisite, to act as a germicide. By varying the time of exposure and the strength of the anticeptic solution, the experiment is repeated, until the minimum time and strength have been ascertained, which are requisite to destroy the bacteria in the material used. Of course each test was verified by careful microscopic examination in order to determine the presence or absence of micro-organisms, and when found, their character and condition.

The first series of experiments which were undertaken more than a year ago were made as follows: The aseptic solution was prepared by boiling several small pieces of meat with a large quantity of water in a glass flask, purified by exposure to the flame of an alcohol lamp. This flask was stopped with a rubber cork perforated by two glass tubes for the convenience of decanting. The tubes were bent downwards and the ends were protected with carbolized gauze. 'The cork and tubes were carefully cleansed beforehand with carbolic acid. The resulting solution, if made with lean meat settles clear; if the meat is fat the solution is rendered cloudy by fine oil drops and must be prepared again. The solution thus in a proper condition was placed under a bell jar where it remained indefinitely aseptic. The combined material is placed in a test tube purified by heat and this is covered by a bell glass similiarly puri- 